\title{
Optimal Replacement Policies for a Multistate Degenerative System with Negligible or Nonnegligible Repair Times
}

\author{
P. Govindaraju \\ Department of Mathematics, Islamiah College \\ Vaniyambadi, India \\ E-mail: govindrajmaths69@gmail.com \\ U. Rizwan \\ Department of Mathematics, Islamiah College \\ Vaniyambadi, India \\ E-mail: rizwanmaths@yahoo.com \\ V. Thangaraj \\ Ramanujan Institute for Advanced Study in Mathematics \\ University of Madras, Chennai, India \\ E-mail: thangarajv@unom.ac.in
}

\begin{abstract}
In this paper, a repairable system of a monotone process model for a one-component multistate degenerative system with $(k+1)$ states $(k$-failure states and one working state) is studied. Also an alternative model, called the Negligible Or Non-Negligible (NONN) repair times introduced by Thangaraj and Rizwan (2001) is incorporated in this model to develop some new repair models. A replacement policy $T$ is adapted by which the system will be replaced whenever the working age of the system reaches $T$; another replacement policy $T$ but with NONN repair times; the $N$ policy, based on the number of failures of the system assuming NONN repair times and a bivariate replacement policy $(T, N)$ under NONN repair times, where $T$ is the working age and $N$ is the number of failures of the system are studied. Furthermore, explicit expressions for the long-run average cost of the above policies are derived. Also the conditions for the existence of univariate optimal replacement policies are derived. Finally, we show that the optimal policy $(T, N)^{*}$ is better than the optimal policy $N^{*}$ or the optimal policy $T^{*}$. We conclude with a conjecture that $N$-policy is the best replacement policy for any deteriorating system among the univariate policies.
\end{abstract}

Keywords: Geometric process, Replacement policy, Renewal reward process, Stochastic orders, NONN repair times

\section{Introduction}

The mathematical theory of reliability has applied itself to problems of life-testing, machine maintenance, replacement, order statistics, etc. Most of the systems are degenerative, because of the ageing effect and accumulated wearing. The successive operating times between failures tend to decrease, while the consecutive repair times after failures tend to increase. That is, the successive operating times are stochastically decreasing, while the consecutive repair times are stochastically increasing. In practice, the repair of a failure system will usually yield a functioning system, its successive operating times are decreasing and finally dying out. For deteriorating systems, this is often the case. On the other hand, the consecutive repair times will be increasing and finally, tend to infinity, that is, finally the system is non-repairable. Thus, it is reasonable to assume that the repair times are, in general, non-negligible. This is referred to as a geometric process, introduced and studied by Lam (1988) in his pioneering work.

In the above models, a system, in general, has only two states, the operating and the failure states; usually referred to as: up and down states. However, in real life situations, a system, in general, may have more than two states. For example, the system constituted by an electronic instrument may break down due to a short circuit or an open circuit, in which case the 
system will have two failure states and one working state. Lam, Zhang and Zheng (2002) have considered an equivalent geometric process model for a multistate degenerative system with $k$-failure states and one working state. Also, they have given an analytic expression for the long-run average cost per unit time using a replacement policy which depends only on the number of failures of the system. However Zhang, Yam and Zuo(2002) have derived the long-run expected profit for the model introduced and studied by Lam, Zhang and Zheng (2002). Further Zhang, Yam and Zuo (2007) have derived an explicit expression for the long-run expected profit for the same model, considering a bivariate replacement policy, which depends either on the working age of the system or the number of repairs of the system. There is always a speculation about the influence of working age of a system. It urges us to consider, in this paper, a replacement policy which depends on the working age of the system for a multistate degenerative system with $k$-failure states and one working state. Further, repair times are generally assumed to be non-decreasing in the case of geometric process. It is not true that the repair times always tend to increase. The repair times usually depend on the type of failure occurred at a specific moment in the system. To overcome this, Thangaraj and Rizwan (2001) have introduced probability for different types of failures of a repairable system and called it NONN repair times. Thus it is reasonable to assume this alternative repair model. Considering this alternative repair model, it is proposed to study some univariate and bivariate optimal replacement policies and desired to compare these policies using a numerical example.

Here a customary summary of known definitions of some basic concepts which are needed for our discussion is outlined below.

Definition 1.1. A random variable $X$ is said to be stochastically smaller than another random variable $Y$, if $P(X>\alpha) \leq$ $P(Y>\alpha)$, for all real $\alpha$. It is denoted by $X \leq_{s t} Y$. Further, a stochastic process $\left\{X_{n}, n=1,2, \ldots\right\}$ is said to be stochastically increasing, if $X_{n} \leq_{s t} X_{n+1}$, for $n=1,2, \ldots$.

The definition of monotone concept for a stochastic process $\left\{X_{n}, n=1,2, \ldots\right\}$ is based on the distributions of $X_{n}$ and $X_{n+1}$, for $n=1,2, \ldots$. Lam(1988) has introduced the geometric process for a simple monotone process.

Definition 1.2. A stochastic process $\left\{X_{n}, n=1,2, \ldots\right\}$ is a geometric process (GP), if there exist a real constant $a>0$ such that $\left\{a^{n-1} X_{n}, n=1,2, \ldots\right\}$ forms a renewal process. The number $a$ is called the ratio of the geometric process.

If $0<a<1$, the GP is stochastically increasing; if $a>1$, the GP is stochastically decreasing and if $a=1$, the GP will reduce to a renewal process.

Definition 1.3. An integer valued random variable $N$ is said to be a stopping time for the sequence of independent random variables $X_{1}, X_{2}, \ldots$, if the event $\{N=n\}$ is independent of $X_{n+1}, X_{n+2}, \ldots$, for all $n=1,2, \ldots$.

Theorem 1.4. Wald's equation. If $X_{1}, X_{2}, \ldots$ are independent and identically distributed random variables having finite expectations and if $N$ is the stopping time for $X_{1}, X_{2}, \ldots$ such that $E[N]<\infty$, then

$$
E\left[\sum_{n=1}^{N} X_{n}\right]=E(N) E\left(X_{1}\right)
$$

Definition 1.5. The T-policy.

It is a policy under which the system will be replaced whenever the working age of the system reaches $T$.

Definition1.6. The $N$-policy.

It is a policy under which the system will be replaced upon the $N$-th failure of the system, since the last replacement.

Definition 1.7. The bivariate $(T, N)$-policy.

It is a policy under which the system will be replaced at the working age $T$ or at the time of $N$-th failure since the last replacement, whichever occurs earlier.

\section{Definition 1.8. NONN Repair times}

If a repair to a system after failure is done in negligible or non-negligible time, then it will be called a model with NONN repair times.

In this case, whenever the system fails, two possibilities may arise: either, the repair takes Negligible time with probability $p$; or Non-Negligible time with probability $1-p$.

The main objective of this paper is to obtain explicit expressions for the long-run average cost per unit time for the maintenance model of a multistate degenerative system with $k$-failure states and one working state under the aforesaid preventive maintenance policies, assuming different repair models.

The rest of the paper is organized as follows: In Section 2, we present the model and derive an expression for the longrun average cost per unit time for this model under $T$-policy. We also derive the conditions for existence of the optimal replacement policy $T^{*}$ (under this model) in this section. In Section 3, we present the alternative repair model and derive an explicit expression for the long-run average cost per unit time for this model. The existence of the optimal replacement policy is also derived in this section. In Section 4, we derive an expression for the long-run average cost for this model 
under $N$-policy. The necessary conditions for the existence of the optimal replacement policy $N^{*}$ are also derived in this section. In Section 5, we derive an expression for the long-run average cost for this model under a bivariate replacement policy $(T, N)$. Comparison between policies have been made in Section 6. A numeric example is given in Section 7, to illustrate the results, developed in this paper. Comparison of numerical result have been carried out in Section 8 . Finally, conclusion is given in Section 9.

\section{The Replacement Policy $T$}

In this section, we introduce and study a $T$-policy for a multistate one component degenerative system. Under the replacement policy $T$, the problem is to determine an optimal replacement policy $T^{*}$ such that the long-run average cost per unit time is minimized.

We consider a monotone process model for a multistate one-component degenerative system and following Lam, Zhang and Zheng (2002), we make the following assumptions. For the sake of readability, we state the same here.

Assumption 2.1. At time $t=0$, a new system is put into field use. Whenever the system fails, it will be repaired. The system will be replaced by an identical new one, some time later.

Assumption 2.2. The system state at time $t$, denoted by $S(t)$ is

$$
S(t)= \begin{cases}0, & \text { if the system is working at time } t \\ i, & \text { if the system is in the } i \text {-th type of failure } \\ & \text { state at time } t, i=1,2, \ldots, k\end{cases}
$$

Thus the state space is $\Omega=\{0,1,2, \ldots, k\}$. If the system fails, then with probability $p_{i}$, the system will be in state $i$, $i=1,2, \ldots, k$ and $\sum_{i=1}^{k} p_{i}=1$.

Assumption 2.3. Let $X_{1}$ be the first operating time. For $n \geq 2$, let $X_{n}$ be the operating time of the system after $(n-1)$-st repair, let $Y_{n}$ be the repair time after the $n$-th failure and $Z$ be the replacement time. Now, denote the time of the $n$-th failure by $t_{n}$. Assume that

$$
\text { and } \begin{aligned}
P\left(X_{1} \leq t\right) & =U(t), \\
P\left(X_{2} \leq t \mid S\left(t_{1}\right)=i\right) & =U\left(a_{i} t\right), \quad i=1,2, \ldots, k .
\end{aligned}
$$

In general, for $j=1,2, \ldots, n-1 ; i_{j}=1,2, \ldots, k$,

$$
P\left(X_{n} \leq t \mid S\left(t_{1}\right)=i_{1}, \ldots, S\left(t_{n-1}\right)=i_{n-1}\right)=U\left(a_{i_{1}} \cdots a_{i_{n-1}} t\right),
$$

where $1 \leq a_{1} \leq a_{2} \leq \cdots \leq a_{k}$. Similarly, assume that

$$
P\left(Y_{1} \leq t \mid S\left(t_{1}\right)=i\right)=V\left(b_{i} t\right), \quad i=1,2, \ldots, k .
$$

In general, for $j=1,2, \ldots, n ; i_{j}=1,2, \ldots, k$,

$$
P\left(Y_{n} \leq t \mid S\left(t_{1}\right)=i_{1}, \ldots, S\left(t_{n}\right)=i_{n}\right)=V\left(b_{i_{1}} \cdots b_{i_{n}} t\right),
$$

where $1 \geq b_{1} \geq b_{2} \geq \cdots \geq b_{k}>0$.

Assumption 2.4. The working age of the system at time $T$ is the cumulative life-time given by

$$
T(t)=\left\{\begin{array}{lr}
t-M_{n}, & L_{n}+M_{n} \leq t<L_{n+1}+M_{n} \\
L_{n+1}, & L_{n+1}+M_{n} \leq t<L_{n+1}+M_{n+1}
\end{array}\right.
$$

where $L_{n}=\sum_{i=1}^{n} X_{i}$ and $M_{n}=\sum_{i=1}^{n} Y_{i}$ and $M_{0}=N_{0}=0$.

Assumption 2.5. Let $r$ be the reward rate per unit time of the system when it is operating and $c$ be the repair cost rate per unit time of the system. Assume further that the replacement cost comprises of two parts: one part is the basic replacement $\operatorname{cost} R$ and the other part is the cost proportional to the length of replacement time $Z$ at rate $c_{p}$.

Assumption 2.6. The replacement policy $T$ is adapted under which the system will be replaced whenever its working age reaches $T$.

Let $E\left(X_{1}\right)=\int_{0}^{\infty} t d U(t)=\lambda$ and $E\left(Y_{1}\right)=\int_{0}^{\infty} t d V(t)=\mu$. Following Lam, Zhang and Zheng (2002), we have $E\left(X_{n}\right)=\frac{\lambda}{a^{n-1}}$, where $a=\left(\sum_{i=1}^{k} \frac{p_{i}}{a_{i}}\right)^{-1}$ and $E\left(Y_{n}\right)=\frac{\mu}{b^{n}}$, where $b=\left(\sum_{i=1}^{k} \frac{p_{i}}{b_{i}}\right)^{-1}$. 
Let $T_{1}$ be the first replacement time and in general for $n \geq 2$, let $T_{n}$ be the time between $(n-1)$-st replacement and $n$-th replacement. Thus the sequence $T_{1}, T_{2}, \ldots$ constitutes a renewal process, while the interarrival times between two consecutive replacements is a renewal cycle. Further, a cycle is completed, if a replacement is done. By the theory of renewal reward process, the long-run average cost per unit time is given by

$$
\begin{aligned}
\mathscr{C}(T) & =\frac{\text { the expected cost incurred in a cycle }}{\text { the expected length of a cycle }} \\
= & \frac{c E\left(\sum_{i=1}^{\eta-1} Y_{i}\right)+R+c_{p} E(Z)-r E\left(\sum_{i=1}^{\eta} X_{i}\right)}{E\left(\sum_{i=1}^{\eta} X_{i}\right)+E\left(\sum_{i=1}^{\eta-1} Y_{i}\right)+E(Z)},
\end{aligned}
$$

where $\eta$ is a random variable denoting the number of failures in time $T$. Since $\eta$ is also a stopping time with respect to the $\sigma$-fields $\left\{\sigma<X_{1}, X_{2}, \ldots, X_{\eta}>, \quad \eta=1,2, \ldots\right\}$, by Wald's equation, we have

$$
E\left(\sum_{i=1}^{\eta} X_{i}\right)=E\left(E\left[\sum_{i=1}^{\eta} X_{i} \mid \eta=n\right]\right)=\lambda \sum_{n=1}^{\infty} \frac{F_{n}(T)}{a^{n-1}} .
$$

where $F_{n}(\cdot)$ is the $n$-fold convolution of $F(\cdot)$ with itself and

$$
E\left(\sum_{i=1}^{\eta-1} Y_{i}\right)=E\left(E\left[\sum_{i=1}^{\eta-1} Y_{i} \mid \eta=n-1\right]\right)=\mu \sum_{n=1}^{\infty} \frac{G_{n}(T)}{b^{n}},
$$

where $G_{n}(\cdot)$ is the $n$-fold convolution of $G(\cdot)$ with itself.

Using equations (2.3) and (2.4), equation (2.2) becomes

$$
\mathscr{C}(T)=\frac{c \mu \sum_{n=1}^{\infty} \frac{G_{n}(T)}{b^{n}}+R+c_{p} \tau-r \lambda \sum_{n=1}^{\infty} \frac{F_{n}(T)}{a^{n-1}}}{\lambda \sum_{n=1}^{\infty} \frac{F_{n}(T)}{a^{n-1}}+\mu \sum_{n=1}^{\infty} \frac{G_{n}(T)}{b^{n}}+\tau}
$$

where $E(Z)=\tau$. Further

$$
\mathscr{C}(T)=\frac{(c+r) \mu \sum_{n=1}^{\infty} \frac{G_{n}(T)}{b^{n}}+R_{1}}{\lambda \sum_{n=1}^{\infty} \frac{F_{n}(T)}{a^{n-1}}+\mu \sum_{n=1}^{\infty} \frac{G_{n}(T)}{b^{n}}+\tau}-r,
$$

where $R_{1}=R+\left(c_{p}+r\right) \tau$. Let $A_{n}=\frac{\mu}{b^{n}}$ and $B_{n}=\frac{\lambda}{a^{n-1}}$. Then $\mathscr{C}(T)$ can be rewritten as

$$
\mathscr{C}(T)=\frac{(c+r) \sum_{n=1}^{\infty} A_{n} G_{n}(T)+R_{1}}{\sum_{n=1}^{\infty} B_{n} F_{n}(T)+\sum_{n=1}^{\infty} A_{n} G_{n}(T)+\tau}-r .
$$

We observe here that $\sum_{n=1}^{\infty} A_{n} G_{n}(T)$ and $\sum_{n=1}^{\infty} B_{n} F_{n}(T)$ converges absolutely. It follows from Mertens (Rudin(1976)) Theorem that

$$
\sum_{n=1}^{\infty} A_{n} G_{n}(T) \sum_{n=1}^{\infty} B_{n} F_{n}(T)=\sum_{n=1}^{\infty} \sum_{k=1}^{n} A_{k} B_{n-k} G_{k}(T) F_{n-k}(T) .
$$

Since the series are uniformly convergent, term-by-term differentiation is applicable. On equating $\mathscr{C}^{\prime}(T)$ to zero, we obtain

$$
\begin{aligned}
(c+r) \sum_{n=1}^{\infty} \sum_{k=1}^{n} A_{k} B_{n-k}\left\{F_{n-k}(T) G_{k}^{\prime}(T)-G_{k}(T) F_{n-k}^{\prime}(T)\right\} & \\
+\tau(c+r) \sum_{n=1}^{\infty} A_{n} G_{n}^{\prime}(T)-R_{1} \sum_{n=1}^{\infty} B_{n} F_{n}^{\prime}(T) & =0 .
\end{aligned}
$$


If $\mathscr{C}^{\prime \prime}(T)>0$, then we must have

$$
(c+r) \sum_{n=1}^{\infty} \sum_{k=1}^{n} A_{k} B_{n-k}\left\{G_{k}^{\prime \prime}(T) F_{n-k}(T)-G_{k}(T) F_{n-k}^{\prime \prime}(T)-R \sum_{n=1}^{\infty} A_{n} G_{n}^{\prime \prime}(T)-R_{1} \sum_{n=1}^{\infty} B_{n} F_{n}^{\prime \prime}(T)>0 .\right.
$$

Thus (2.8) gives $T^{*}$ for which $\mathscr{C}\left(T^{*}\right)$ is minimum. Summarizing the above facts, we have the following theorem.

Theorem 2.1. The long-run average cost per unit per unit time, $C(T)$ given by (2.5) for the monotone process model for a multistate one-component system under T-policy is minimum, if (2.8) and (2.9) hold.

Moreover, the minimization procedure can also be done by numerical method.

\section{The Replacement Policy $T$ with NONN repair times}

In this section, we introduce and study a $T$-policy for a multistate one-component degenerative system with NONN repair times. Under the replacement policy $T$, the problem is to determine an optimal replacement policy $T^{*}$ such that the long-run average cost per unit time is minimized.

We consider a monotone process model for a multistate one-component degenerative system and make the following assumptions:

Assumptions 3.1, 3.2, 3.4, 3.5 are the same as Assumptions 2.1, 2.2, 2.4 and 2.5.

Assumption 3.3. Let $X_{1}$ be the first operating time. For $n \geq 2$, let $X_{n}$ be the operating time of the system after $(n-1)$-st repair, let $\xi_{n}$ be the repair time after the $n$-th failure and $Z$ be the replacement time. Now, denote the time of the $n$-th failure by $t_{n}$.

Assumption 3.6. The survival times $\left(X_{i}\right)$ and the NONN repair times $\left(\xi_{i}\right)$ and the replacement time $Z$, for $i=1,2, \ldots$ are independent.

Assumption 3.7. The replacement policy $T$ is adapted under which the system will be replaced whenever its working age reaches $T$.

Therefore

$$
\begin{aligned}
E\left(\xi_{n}\right) & =E\left(Y_{n}\right) P\left(Y_{n}>0\right)+1 P\left(Y_{n}=0\right) \\
& =\frac{\mu}{b^{n-1}}(1-p)+p
\end{aligned}
$$

Let $T_{1}$ be the first replacement time and in general for $n \geq 2$, let $T_{n}$ be the time between $(n-1)$-st replacement and $n$-th replacement. Thus the sequence $T_{1}, T_{2}, \ldots$ constitutes a renewal process. Further, a cycle is completed, if a replacement is done. By the theory of renewal reward process, the long-run average cost per ut time is given by

$$
\begin{aligned}
\mathscr{C}(T) & =\frac{\text { the expected cost incurred in a cycle }}{\text { the expected length of a cycle }} \\
& =\frac{c E\left(\sum_{n=1}^{\eta-1} \xi_{n}\right)+R+c_{p} E(Z)-r E\left(\sum_{n=1}^{\eta} X_{n}\right)}{E\left(\sum_{n=1}^{\eta} X_{n}\right)+E\left(\sum_{n=1}^{\eta-1} \xi_{n}\right)+E(Z)},
\end{aligned}
$$

where $\eta$ is a random variable denoting the number of failures in time $T$. Since $\eta$ is also a stopping time with respect to the $\sigma$-fields $\left\{\sigma<X_{1}, X_{2}, \ldots, X_{\eta}>, \quad \eta=1,2, \ldots\right\}$, by Wald's equation,

we have

$$
E\left(\sum_{n=1}^{\eta} X_{n}\right)=\lambda \sum_{n=1}^{\infty} \frac{F_{n}(T)}{a^{n-1}}
$$

where $F_{n}(\cdot)$ is the $n$-fold convolution of $F(\cdot)$ with itself.

Consider

$$
\begin{aligned}
E\left(\sum_{n=1}^{\eta-1} \xi_{n}\right) & =E\left[E\left(\sum_{n=1}^{\eta-1} E\left(\xi_{n}\right) \mid \eta\right)\right] \\
& =\sum_{n=1}^{\infty}\left(\frac{\mu(1-p)}{b^{n-1}}+p\right) G_{n}(T),
\end{aligned}
$$


where $G_{n}(\cdot)$ denotes the $n$-fold convolution of $G(\cdot)$.

Using equations (3.2) and (3.3), equation (3.1) becomes

$$
\mathscr{C}(T)=\frac{c \sum_{n=1}^{\infty}\left(\frac{\mu(1-p)}{b^{n-1}}+p\right) G_{n}(T)+R+c_{p} \tau-r \lambda \sum_{n=1}^{\infty} \frac{F_{n}(T)}{a^{n-1}}}{\lambda \sum_{n=1}^{\infty} \frac{F_{n}(T)}{a^{n-1}}+\sum_{n=1}^{\infty}\left(\frac{\mu(1-p)}{b^{n-1}}+p\right) G_{n}(T)+\tau},
$$

where $E(Z)=\tau$. Further

$$
\mathscr{C}(T)=\frac{(c+r) \sum_{n=1}^{\infty}\left(\frac{\mu(1-p)}{b^{n-1}}+p\right) G_{n}(T)+R_{1}}{\lambda \sum_{n=1}^{\infty} \frac{F_{n}(T)}{a^{n-1}}+\sum_{n=1}^{\infty}\left(\frac{\mu(1-p)}{b^{n-1}}+p\right) G_{n}(T)+\tau}-r
$$

where $R_{1}=R+\left(c_{p}+r\right) \tau$. Let

$$
A_{n}=\frac{\lambda}{a^{n-1}} \quad \text { and } \quad B_{n}=\frac{\mu(1-p)}{b^{n-1}}+p .
$$

Then $\mathscr{C}(T)$ can be rewritten as

$$
\mathscr{C}(T)=\frac{(c+r) \sum_{n=1}^{\infty} B_{n} G_{n}(T)+R_{1}}{\sum_{n=1}^{\infty} A_{n} F_{n}(T)+\sum_{n=1}^{\infty} B_{n} G_{n}(T)+\tau}-r .
$$

On equating $\mathscr{C}^{\prime}(T)$ to zero, we obtain

$$
(c+r) \sum_{n=1}^{\infty} \sum_{k=1}^{n} B_{k} A_{n-k}\left\{F_{n-k}(T) G_{k}^{\prime}(T)-G_{k}(T) F_{n-k}^{\prime}(T)\right\}+\tau(c+r) \sum_{n=1}^{\infty} B_{n} G_{n}^{\prime}(T)-R_{1} \sum_{n=1}^{\infty} A_{n} F_{n}^{\prime}(T)=0 .
$$

If $\mathscr{C}^{\prime \prime}(T)>0$, then we must have

$$
(c+r) \sum_{n=1}^{\infty} \sum_{k=1}^{n} B_{k} A_{n-k}\left[G_{k}^{\prime \prime}(T) F_{n-k}(T)-G_{k}(T) F_{n-k}^{\prime \prime}(T)-R_{1} \sum_{n=1}^{\infty}\left[A_{n} F_{n}^{\prime \prime}(T)+B_{n} G_{n}^{\prime \prime}(T)>0 .\right.\right.
$$

For $\mathscr{C}(T)$ to attain mimum, $\mathscr{C}^{\prime}(T)=0$ and $\mathscr{C}^{\prime \prime}(T)>0$. Thus (3.7) gives $T^{*}$ for which $\mathscr{C}\left(T^{*}\right)$ is mimum. Summarizing the above facts, we have the following theorem.

Theorem 1 The long-run average cost per ut per ut time, $\mathscr{C}(T)$ given by (3.4) for the monotone process alternative repair model of a multistate one-component system under T-policy with NONN repair times is mimum, if (3.7) and (3.8) hold.

\section{Remarks.}

(i) When $p=0$, that is when the repair times non-negligible, equation (3.4) reduces to

$$
\mathscr{C}(T)=\frac{c \mu \sum_{n=1}^{\infty} \frac{G_{n}(T)}{b^{n-1}}+R+c_{p} \tau-r \lambda \sum_{n=1}^{\infty} \frac{F_{n}(T)}{a^{n-1}}}{\lambda \sum_{n=1}^{\infty} \frac{F_{n}(T)}{a^{n-1}}+\mu \sum_{n=1}^{\infty} \frac{G_{n}(T)}{b^{n-1}}+\tau} .
$$

(ii) When $p=1$, that is, when the repair times are negligible, equation(3.4) reduces to

$$
\mathscr{C}(T)=\frac{c \sum_{n=1}^{\infty} G_{n}(T)+R+c_{p} \tau-r \lambda \sum_{n=1}^{\infty} \frac{F_{n}(T)}{a^{n-1}}}{\lambda \sum_{n=1}^{\infty} \frac{F_{n}(T)}{a^{n-1}}+\sum_{n=1}^{\infty} G_{n}(T)+\tau} .
$$




\section{The Replacement Policy $N$ with NONN repair times}

In this section, we study the alternative repair model introduced in the previous section for the maintenance problem of a repairable system and we use the $N$ policy with NONN repair times. Under the replacement policy $N$, the problem is to determine an optimal $N^{*}$ such that the long-run average cost per unit time is minimized. We make the following assumptions :

Assumptions 4.1 to 4.6 are the same as assumptions 3.1 to 3.6 .

Assumption 4.7. A replacement policy $N$ with NONN repair times is adapted. By applying the replacement policy $N$, the system will be replaced by an identical new one at the time following the $N$-th failure. The replacement time is a random variable $Z$ with $E(Z)=\tau$.

Then by the renewal reward theorem, the long-run average cost per unit time under the replacement policy $N$ with NONN repair times is given by

$$
\begin{aligned}
\mathscr{C}(N) & =\frac{\text { the expected cost incurred in a cycle }}{\text { the expected length of a cycle }} \\
& =\frac{c E\left(\sum_{n=1}^{N-1} \xi_{n}\right)+R+c_{p} E(Z)-r E\left(\sum_{n=1}^{N} X_{n}\right)}{E\left(\sum_{n=1}^{N} X_{n}\right)+E\left(\sum_{n=1}^{N-1} \xi_{n}\right)+E(Z)} \\
& =\frac{c \sum_{n=1}^{N-1} E\left(\xi_{n}\right)-r \sum_{n=1}^{N} E\left(X_{n}\right)+R+c_{p} E(Z)}{\sum_{n=1}^{N} E\left(X_{n}\right)+\sum_{n=1}^{N-1} E\left(\xi_{n}\right)+E(Z)}
\end{aligned}
$$

Now

$$
\begin{aligned}
E\left(\sum_{n=1}^{N-1} \xi_{n}\right) & =\sum_{n=1}^{N-1}\left[E\left(Y_{n}\right) P\left(Y_{n}>0\right)+1 P\left(Y_{n}=0\right)\right] \\
& =\sum_{n=1}^{N-1}\left(\frac{\mu}{b^{n}}(1-p)+p\right)
\end{aligned}
$$

and

$$
E\left(\sum_{n=1}^{N} X_{n}\right)=\sum_{n=1}^{N} \frac{\lambda}{a^{n-1}}
$$

On substituting (4.2) and (4.3), equation (4.1) becones

$$
\mathscr{C}(N)=\frac{(c+r) \sum_{n=1}^{N-1}\left(\frac{\mu}{b^{n}}(1-p)+p\right)+R_{1}}{\lambda \sum_{n=1}^{N} \frac{1}{a^{n-1}}+\sum_{n=1}^{N-1}\left(\frac{\mu}{b^{n}}(1-p)+p\right)+\tau}-r
$$

where $R_{1}=R+\left(c_{p}+r\right) \tau$.

In order to minimize $\mathscr{C}(N)$, we define $\mathscr{A}(N)$ and note that minimizing $\mathscr{C}(N)$ is equivalent to minimizing $\mathscr{A}(N)$.

$$
\mathscr{A}(N)=\frac{(c+r) \sum_{i=1}^{N-1}\left(\frac{\mu}{b^{n}}(1-p)+p\right)+R_{1}}{\lambda \sum_{n=1}^{N} \frac{1}{a^{n-1}}+\sum_{n=1}^{N-1}\left(\frac{\mu}{b^{n}}(1-p)+p\right)+\tau} .
$$


We now find the difference between $\mathscr{A}(N+1)$ and $\mathscr{A}(N)$.

$$
\mathscr{A}(N+1)-\mathscr{A}(N)=\frac{\left[\begin{array}{r}
(c+r)\left(\frac{\mu}{b^{N}}(1-p)+p\right)\left(\tau+\sum_{n=1}^{N} \frac{\lambda}{a^{n-1}}\right)-R_{1}\left(\frac{\lambda}{a^{N-2}}+\left(\frac{\mu}{b^{N}}(1-p)+p\right)\right) \\
-(c+r) \frac{\lambda}{a^{N}} \sum_{n=1}^{N-1}\left(\frac{\mu}{b^{n}}(1-p)+p\right)
\end{array}\right]}{\left[\sum_{n=1}^{N+1} \frac{\lambda}{a^{n-1}}+\sum_{n=1}^{N}\left(\frac{\mu}{b^{n}}(1-p)+p\right)+\tau\right]\left[\sum_{n=1}^{N} \frac{\lambda}{a^{n-1}}+\sum_{n=1}^{N-1}\left(\frac{\mu}{b^{n}}(1-p)+p\right)+\tau\right]} .
$$

Here the denominator of the right side above term is positive. Define

$$
\mathscr{B}(N)=\frac{R_{1}\left[\frac{\lambda}{a^{N-2}}+\left(\frac{\mu}{b^{N}}(1-p)+p\right)\right]}{(c+r)\left[\left(\frac{\mu}{b^{n}}(1-p)+p\right) \sum_{n=1}^{N} \frac{\lambda}{a^{n-1}}-\frac{\lambda}{a^{N}}\left(\frac{\mu}{b^{N}}(1-p)+p\right)+\left(\frac{\mu}{b^{n}}(1-p)+p\right) \tau\right]}
$$

Since the denominator of $\mathscr{A}(N+1)-\mathscr{A}(N)$ is always positive, the sign of $\mathscr{A}(N+1)-\mathscr{A}(N)$ is the same as the sign of its numerator. Concomitantly, we have the following.

Lemma $1 \mathscr{A}(N)$ is either non-decreasing (or non-increasing) in $N$ if and only if $\mathscr{B}(N) \geq 1(\leq 1)$

Lemma 2 For the model described in this section, under assumptions 4.1 to 4.7, we have,

$$
\mathscr{B}(N) \text { is non-decreasing in Nif and only if } b^{N+1} p(1-a) \geq \mu(1-p)(a-b)
$$

Proof. Here

$$
\begin{aligned}
\mathscr{B}(N+1)-\mathscr{B}(N)= & {\left[\frac{\lambda}{a^{N-1}}+\frac{\mu}{b^{N+1}}(1-p)+p\right]\left[\left(\frac{\mu}{b^{N}}(1-p)+p\right) \sum_{n=1}^{N} \frac{\lambda}{a^{n-1}}\right.} \\
& \left.-\frac{\lambda}{b^{N}} \sum_{n=1}^{N-1}\left(\frac{\mu}{b^{n}}(1-p)+p\right)+\left(\frac{\mu}{b^{N}}(1-p)+p\right) \tau\right]-\frac{\lambda}{a^{N-2}} \\
& +\left(\frac{\mu}{b^{N}}(1-p)+p\right)\left(\frac{\mu}{b^{N+1}}(1-p)+p\right) \sum_{n=1}^{N+1} \frac{\lambda}{a^{n-1}} \\
& -\frac{\lambda}{b^{N+1}} \sum_{n=1}^{N}\left(\frac{\mu}{b^{n}}(1-p)+p\right)+\left(\frac{\mu}{b^{N+1}}(1-p)+p\right) \tau
\end{aligned}
$$

Note here that $\mathscr{B}(N+1)-\mathscr{B}(N) \geq 0$, that is, $\mathscr{B}(N)$ is non-decreasing in $N$ if and only if

$$
\begin{gathered}
\left(\frac{\mu}{b^{N}}(1-p)+p\right)-a\left(\frac{\mu}{b^{N+1}}(1-p)+p\right) \geq 0 \\
\mu b(1-p)+b^{N+1} p-a \mu(1-p)-a b^{N+1} p \geq 0,
\end{gathered}
$$

which on simplification yields (4.5)

Moreover the minimization procedure can be done by analytical or numerical methods.

Theorem 2 For the model described in this section under assumptions 4.1 to 4.7, an optimal replacement policy $N^{*}$ can be determined by

$$
N^{*}=\min \{N: \mathscr{B}(N) \geq 1\}
$$

Remark. If $N=1$, then

$$
\mathscr{B}(1)=\frac{(c+r)[\mu(1-p)+p]\left(\lambda_{1}+\tau\right)}{(R+r \tau)\left(\lambda_{2}+\mu(1-p)\right)}
$$

If $\mathscr{B}(1) \geq 1$, then $N^{*}=1$. This means that an optimal replacement policy is to be replace the system immediately whenever it fails. If $\mathscr{B}(\infty)$ exist and $\mathscr{B}(\infty) \leq 1$, then $N^{*}=\infty$. This means the optimal policy is to continually repair the system as it ages without ever replacing it. 
We now give the expression for the long-run average cost per unit time derived for a multistate one-component system under $N$-policy by Lam, Zhang and Zheng (2002) which is needed for comparison of policies.

$$
\mathscr{C}(N)=\frac{c \mu \sum_{i=1}^{N-1} \frac{1}{b^{i}}+R+c_{p} \tau-r \lambda \sum_{i=1}^{N} \frac{1}{a^{i-1}}}{\lambda \sum_{i=1}^{N} \frac{1}{a^{i-1}}+\mu \sum_{i=1}^{N-1} \frac{1}{b^{i}}+\tau} .
$$

\section{The Bivaritae Policy $(T, N)$ with NONN repair times}

In this section, we study a bivariate policy $(T, N)$ with NONN repair times under which the system is replaced at working age $T$ or at the time following the $N$-th failure, whichever occurs first. The problem is to choose an optimal replacement policy $(T, N)^{*}$ such that the long-run average cost per unit time is minimized. We make the following assumptions. Assumptions 5.1 to 5.5 are the same as Assumptions 2.1 to 2.5.

Assumption 5.6. The replacement policy $(T, N)$ is used. Let $T_{1}$ be the first replacement time and in general for $n \geq 2$, let $T_{n}$ be the time between the $(n-1)$-st replacement and the $n$-th replacement. Then the sequence $\left\{T_{n}, n=1,2, \ldots\right\}$ forms a renewal process. Therefore, the inter arrival times between two consecutive replacements is a renewal cycle.

Let $\mathscr{C}(T, N)$ be the long-run average cost per unit time under the bivariate replacement policy $(T, N)$. Then according to the renewal reward theorem, the long-run average cost per unit time is given by

$$
\begin{aligned}
\mathscr{C}(T, N) & =\frac{\text { the expected cost incurred in a cycle }}{\text { the expected length of a cycle }} \\
& =\frac{E\left\{\left(c \sum_{n=1}^{\eta} \xi_{n}-r T\right) \chi_{\left(L_{N}>T\right)}\right\}+c_{p} E(Z)+E\left\{\left(c \sum_{n=1}^{N-1} \xi_{n}-r \sum_{n=1}^{N} X_{n}\right) \chi_{\left(L_{N} \leq T\right)}\right\}+R}{E(W)},
\end{aligned}
$$

where $\eta$ is a random variable denoting the number of failures before the working age of the system reaches $T, W$ is the length of a cycle and

$$
\chi_{A}= \begin{cases}1, & \text { if event } A \text { occurs } \\ 0, & \text { if event } A \text { does not occur }\end{cases}
$$

denotes the indicator function. Therefore $\eta=0,1, \ldots, N-1$.

The length of the cycle under the replacement policy $(T, N)$ is

$$
W=\left(T+\sum_{n=1}^{\eta} \xi_{n}\right) \chi_{\left(L_{N}>T\right)}+\left(\sum_{n=1}^{N} X_{n}+\sum_{n=1}^{N-1} \xi_{n}\right) \chi_{\left(L_{N} \leq T\right)}+Z
$$

where $\eta=0,1,2, \ldots, N-1$ is the number of failures before the working age of the system exceeds $T$. Now

$$
\begin{aligned}
E\left[\left(\sum_{n=1}^{N} X_{n}\right) \chi_{\left(L_{N} \leq T\right)}\right] & =E\left\{E\left[\left(\sum_{n=1}^{N} X_{n}\right) \chi_{\left(L_{N} \leq T\right)} \mid L_{N}\right]\right\} \\
& =\int_{0}^{T} E\left(\sum_{n=1}^{N} X_{n} \mid L_{N}=u\right) d F_{N}(u) \\
& =\int_{0}^{T} u d F_{N}(u)
\end{aligned}
$$


and

$$
\begin{aligned}
E\left[\left(\sum_{n=1}^{N-1} \xi_{n}\right) \chi_{\left(L_{N} \leq T\right)}\right] & =E\left\{E\left[\left(\sum_{n=1}^{N-1} \xi_{n}\right) \chi_{\left(L_{N} \leq T\right)} \mid L_{N}\right]\right\} \\
& =\int_{0}^{T} E\left[\sum_{n=1}^{N-1} \xi_{n} \mid L_{N}=u\right) d F_{N}(u) \\
& =\int_{0}^{T}\left(\sum_{n=1}^{N-1} E\left(\xi_{n}\right)\right) d F_{N}(u) \\
& =\int_{0}^{T} \sum_{n=1}^{N-1}\left(\frac{\mu}{b^{n-1}}(1-p)+p\right) d F_{N}(u) \\
& =\sum_{n=1}^{N-1}\left(\frac{\mu}{b^{n-1}}(1-p)+p\right) \int_{0}^{T} d F_{N}(u) \\
& =\sum_{n=1}^{N-1}\left(\frac{\mu}{b^{n-1}}(1-p)+p\right) F_{N}(T)
\end{aligned}
$$

Further

$$
\begin{aligned}
E(W)=E\left[\left(T+\sum_{n=1}^{\eta} \xi_{n}\right) \chi_{\left(L_{N}>T\right)}\right] & +E\left[\left(\sum_{n=1}^{N} X_{n}+\sum_{n=1}^{N-1} \xi_{n}\right) \chi_{\left(L_{N} \leq T\right)}\right]+E(Z) \\
= & E\left[\begin{array}{l}
\left.T \chi_{\left(L_{N}>T\right)}\right]+E\left[\left(\sum_{n=1}^{\eta} \xi_{n}\right) \chi_{\left(L_{N}>T\right)}\right] \\
\left.+E\left\{E\left[\sum_{n=1}^{N} X_{n}+\sum_{n=1}^{N-1} \xi_{n}\right) \chi_{\left(L_{N} \leq T\right)} \mid L_{N}\right]\right\}+E(Z)
\end{array}\right. \\
= & T \bar{F}_{N}(T)+E\left[\sum_{n=1}^{N-1} \xi_{n} \chi_{\left(L_{i}<T<L_{N}\right)}\right] \\
& \quad+\int_{0}^{T} u d F_{N}(u)+\sum_{n=1}^{N-1}\left(\frac{\mu}{b^{n-1}}(1-p)+p\right) F_{N}(T)+\tau,
\end{aligned}
$$

where $b$ is as given in section 2. Let $W_{N-n}=\sum_{j=n+1}^{N} X_{j}$. Then $L_{N}=L_{n}+W_{N-n}$. Moreover $L_{n}$ and $W_{N-n}$ are independent and

$$
H_{N-n}(t)=\int_{0}^{\infty} H_{N-1-n}(a(t-y)) d H(y)
$$

where $H_{N-1-n}(t)$ is the distribution of $\sum_{j=n+1}^{N} X_{j}$ and $a$ is as given in section 2. Since the distribution function of $X_{n+1}$ is $H(t)=F\left(a^{n} t\right)$, equation (5.5) can be written, by induction, as $H_{N-n}(t)=F_{N-n}\left(a^{n} t\right)$. Now

$$
\begin{aligned}
E\left[\chi_{\left(L_{n}<T<L_{N}\right)}\right] & =P\left(L_{n}<T<L_{n}+W_{N-n}\right) \\
& =\int_{0}^{T} \int_{T-u}^{\infty} d H_{N-i}(t) d F_{n}(u) \\
& =\int_{0}^{T} \bar{F}_{N-n}\left(a^{n}(T-u)\right) d F_{n}(u)
\end{aligned}
$$


so that equation (5.4) becomes

$$
\begin{array}{r}
E(W)=T \bar{F}_{N}(T)+\sum_{n=1}^{N-1}\left(\frac{\mu}{b^{n-1}}(1-p)+p\right) \int_{0}^{T} \bar{F}_{N-n}\left(a^{n}(T-u)\right) d F_{n}(u) \\
\quad+\int_{0}^{T} u d F_{N}(u)+\sum_{n=1}^{N-1}\left(\frac{\mu}{b^{n-1}}(1-p)+p\right) F_{N}(t)+\tau \\
=\int_{0}^{T} \bar{F}_{N}(u) d u+\sum_{n=1}^{N-1}\left(\frac{\mu}{b^{n-1}}(1-p)+p\right) F_{N}(T) \\
+\sum_{n=1}^{N-1}\left(\frac{\mu}{b^{n-1}}(1-p)+p\right) \int_{0}^{T} \bar{F}_{N-n}\left(a^{n}(T-u)\right) d F_{n}(u)+\tau .
\end{array}
$$

Equation (5.1) now becomes

$$
\begin{aligned}
& \mathscr{C}(T, N)=\frac{\left[\begin{array}{l}
c \sum_{n=1}^{N-1}\left(\frac{\mu}{b^{n-1}}(1-p)+p\right) \int_{0}^{T} \bar{F}_{N-n}\left(a^{n}(T-u)\right) d F_{n}(u)-r T \bar{F}_{N}(T) \\
+\int_{0}^{T} c \sum_{n=1}^{N-1}\left(\frac{\mu}{b^{n-1}}(1-p)+p\right) d F_{N}(u)-r \int_{0}^{T} u d F_{N}(u)+R+c_{p} \tau
\end{array}\right]}{\left[\begin{array}{l}
\int_{0}^{T} \bar{F}_{N}(u) d u+\sum_{n=1}^{N-1}\left(\frac{\mu}{b^{n-1}}(1-p)+p\right) F_{N}(T) \\
+\sum_{n=1}^{N-1}\left(\frac{\mu}{b^{n-1}}(1-p)+p\right) \int_{0}^{T} \bar{F}_{N-n}\left(a^{n}(T-u)\right) d F_{n}(u)+\tau
\end{array}\right]} \\
& =\frac{\left[\begin{array}{l}
c\left\{\sum_{n=1}^{N-1}\left(\frac{\mu}{b^{n-1}}(1-p)+p\right) F_{N}(T)\right. \\
\left.+\sum_{n=1}^{N-1}\left(\frac{\mu}{b^{n-1}}(1-p)+p\right) \int_{0}^{T} \bar{F}_{N-n}\left(a^{n}(T-u)\right) d F_{n}(u)\right\} \\
+R+c_{p} \tau-r \int_{0}^{T} \bar{F}_{N}(u) d u
\end{array}\right]}{\left[\begin{array}{l}
\int_{0}^{T-1} \bar{F}_{N}(u) d u+\sum_{n=1}^{N}\left(\frac{\mu}{b^{n-1}}(1-p)+p\right) F_{N}(T) \\
+\sum_{n=1}^{N-1}\left(\frac{\mu}{b^{n-1}}(1-p)+p\right) \int_{0}^{T} \bar{F}_{N-n}\left(a^{n}(T-u)\right) d F_{n}(u)+\tau
\end{array}\right]}
\end{aligned}
$$

This is a bivariate function. Obviously, when $N$ is fixed, $\mathscr{C}(T, N)$ is a function of $T$. For fixed $N=m$, it can be written as

$$
\mathscr{C}(T, N)=\mathscr{C}_{m}(T), \quad m=1,2, \ldots
$$

Thus, for a fixed $m$, we can find $T_{m}^{*}$ by analytical or numerical methods such that $\mathscr{C}_{m}\left(T_{m}^{*}\right)$ is minimized. That is, when $N=$ $1,2, \ldots, m, \ldots$, we can find $T_{1}^{*}, T_{2}^{*}, \ldots, T_{m}^{*}, \ldots$, respectively, such that the corresponding $\mathscr{C}_{1}\left(T_{1}^{*}\right), \mathscr{C}_{2}\left(T_{2}^{*}\right), \ldots, \mathscr{C}_{m}\left(T_{m}^{*}\right), \ldots$ are minimized.

Because the total lifetime of a multistate degenerative system is limited, the minimum of the long-run average cost per unit time exists. So we can determine the minimum of the long-run average cost per unit time based on $\mathscr{C}_{1}\left(T_{1}^{*}\right), \mathscr{C}_{2}\left(T_{2}^{*}\right), \ldots, \mathscr{C}_{m}\left(T_{m}^{*}\right), \ldots$

For example, if the minimum is denoted by $\mathscr{C}_{n}\left(T_{n}^{*}\right)$, we obtain the bivariate optimal replacement policy $(T, N)^{*}$ such that

$$
\mathscr{C}\left((T, N)^{*}\right)=\min _{N} \mathscr{C}_{N}\left(T_{N}^{*}\right)
$$

\section{Remarks.}


(i) When $p=0$, equation (5.6) reduces to

$$
\mathscr{C}(T, N)=\frac{\left[\begin{array}{c}
c \sum_{n=1}^{N-1}\left(\frac{\mu}{b^{n-1}}\right) F_{N}(T)+\sum_{n=1}^{N-1}\left(\frac{\mu}{b^{n-1}}\right) \int_{0}^{T} \bar{F}_{N-n}\left(a^{n}(T-u)\right) d F_{n}(u) \\
+R+c_{p} \tau-r \int_{0}^{T} \bar{F}_{N}(u) d u
\end{array}\right]}{\int_{0}^{T} \bar{F}_{N}(u) d u+\sum_{n=1}^{N-1}\left(\frac{\mu}{b^{n-1}}\right) F_{N}(T) \sum_{n=1}^{N-1}\left(\frac{\mu}{b^{n-1}}\right) \int_{0}^{T} \bar{F}_{N-n}\left(a^{n}(T-u)\right) d F_{n}(u)+\tau},
$$

and in this case (5.6) reduces to $\mathscr{C}(T, N)$ given by (10) of Zhang, Yam and Zuo (2007).

(ii) When $p=1$, equation (5.6) reduces to

$$
\mathscr{C}(T, N)=\frac{c(N-1) F_{N}(T)+\sum_{n=1}^{N-1}(N-1) \int_{0}^{T} \bar{F}_{N-n}\left(a^{n}(T-u)\right) d F_{n}(u)+R+c_{p} \tau-r \int_{0}^{T} \bar{F}_{N}(u) d u}{\int_{0}^{T} \bar{F}_{N}(u) d u+(N-1) F_{N}(T)(N-1) \int_{0}^{T} \bar{F}_{N-n}\left(a^{n}(T-u)\right) d F_{n}(u)+\tau},
$$

\section{Comparison of Policies}

6.1 Comparison of $T$-policy with $N$-policy.

If for any $T>0$,

$$
\sum_{n=1}^{\infty} \frac{F_{n}(T)}{a^{n-2}}=\infty,
$$

then $\mathscr{C}(T)=c$. Therefore

$$
\mathscr{C}\left(N^{*}\right)=\min _{N} \mathscr{C}(N) \leq \mathscr{C}(\infty) \equiv c .
$$

It follows that $N$-policy is better than $T$-policy.

6.2 Comparison of the Bivariate policy $(T, N)$ with the univariate $T$-policy and $N$-policy.

When $T \rightarrow \infty$,

$$
\lim _{T \rightarrow \infty} \mathscr{C}(T, N)=\frac{c \mu \sum_{i=1}^{N-1} \frac{1}{b^{i}}-\lambda r \sum_{i=1}^{N} \frac{1}{a^{i-1}}+R+c_{p} \tau}{\lambda \sum_{i=1}^{N} \frac{1}{a^{i-1}}+\mu \sum_{i=1}^{N-1} \frac{1}{b^{i}}+\tau}
$$

If the constants $c, r, R, c_{p}$, and the expected values $\lambda, \mu$, and $\tau$ are the same as in Lam, Zhang and Zheng (2002)'s model, then

$$
\begin{aligned}
\mathscr{C}\left((T, N)^{*}\right) & =\min _{N} \mathscr{C}_{N}\left(T_{N}^{*}\right)=\min _{N}\left[\min _{T} \mathscr{C}(T, N)\right] \\
& \leq \min _{N}[\mathscr{C}(\infty, N)]=\mathscr{C}\left(N^{*}\right) .
\end{aligned}
$$

Hence the bivariate optimal replacement policy $(T, N)^{*}$ is better than the univariate optimal replacement policies $T^{*}$ and $N^{*}$.

The above analysis leads us to conclude that a bivariate optimal replacement policy for a multistate degenerative system is still better than the univariate optimal replacement policies for the same system. 


\section{Numerical Examples}

We consider a numerical example to demonstrate the models and methodology developed in this paper. Consider a degenerative simple repairable system with three states including two failure states and one working state, that is, $k=2$. Assume that $p_{1}=0.495, p_{2}=0.505 ; a_{1}=1.005, a_{2}=1.1 ; b_{1}=0.95$ and $b_{2}=0.85$ so that $p_{1}+p_{2}=1,1<a_{1}<a_{2}$ and $1>b_{1}>b_{2}$. Then $a=\left(\frac{p_{1}}{a_{1}}+\frac{p_{2}}{a_{2}}\right)^{-1}=1.048$ and $b=\left(\frac{p_{1}}{b_{1}}+\frac{p_{2}}{b_{2}}\right)^{-1}=0.897$. Let $\lambda=400, \mu=24, c=8 r=600$, $R=50000, c_{p}=3, \tau=12$. Here $\lambda$ and $\mu$ are in time units and $c, r, R, c_{p}$ and $\tau$ are in monetary units. Assuming these values, we now calculate the long run average cost for the two distinct replacement models developed in this paper in the following cases.

\section{Case(I): The $N$-Policy with NNON RePair times}

In this case, using equation (4.4), overpassing numerical calculations, we arrive at $N^{*}=10$ when $p=0$ and $p=0.3$. However, the long-run average cost decreases from 12.9169 to 10.8856 monetary units, as $p$ increase from 0 to 0.3 . For other values of $p$, the corresponding values of $\mathscr{C}(N)$ in equation (4.4) are given in Table 1.

Note here that as the probability $p$ of the NONN repair times increases from 0 to 0.9 , the optimal value $N^{*}$ that minimizes $\mathscr{C}(N)$ given in (4.4) increases from $N^{*}=10$ to $N^{*}=14$, whereas the long-run average cost $\mathscr{C}(N)$ decreases from 12.9169 monetary units to -3.4846 monetary units. However, if $p$ is taken as 1 , that is, when the repair times are non-negligible, then the value of $N^{*}$ is found to be 44 with the corresponding long-run average cost -22.4521 monetary units. Further, for each value of $p$, there is a unique optimal replacement policy $N^{*}$ which minimizes $\mathscr{C}(N)$ given in (4.4). Here $\mathscr{C}\left(N^{*}\right)$ is the unique minimum of the average cost. These are plotted in Fig. 1.

\section{Case(II): The bivariate $(T, N)$-POlicy With NNON Repair times}

Assume that the distribution function of $X_{n}$ is exponential, that is,

$$
\begin{aligned}
F_{n}(t) & =F_{n}\left(a^{n-1} t\right) \\
& =1-\exp \left(-\frac{a^{n-1} t}{\lambda}\right) ; t \geq 0 ; \quad \frac{1}{\lambda}>0 ; \quad a \geq 1 \text { and } n=1,2, \ldots
\end{aligned}
$$

The density function of the sum $\sum_{i=1}^{n} X_{i}$ of the random variables $X_{1}, X_{2}, \ldots, X_{n}$ having exponential distribution and following an increasing geometric process is given by

$$
h_{n}(t)=(-1)^{n-1} \frac{a^{\frac{n(n-1)}{2}}}{\lambda} \sum_{i=1}^{n} \frac{\exp \left(-\frac{a^{n-1} t}{\lambda}\right)}{\prod_{\substack{j=1 \\ i \neq j}}^{n}\left(a^{i-1}-a^{j-1}\right)} .
$$

The distribution function of $\sum_{i=1}^{n} X_{i}$, in this case, is given by

$$
H_{n}(t)=1-\sum_{i=1}^{n}\left[\prod_{\substack{j=1 \\ i \neq j}}^{n} \frac{a^{j-1}}{a^{i-1}-a^{j-1}}\right] \exp \left(-\frac{a^{n-1} t}{\lambda}\right), \text { for } t \geq 0 .
$$

Equation (5.6) becomes

$$
\mathscr{C}(T, N)=\frac{c \sum_{n=1}^{N-1}\left(\frac{\mu}{b^{n-1}}(1-p)+p\right) H_{n}(T)+R+c_{p} \tau-r \int_{0}^{T} \bar{F}_{N}(u) d u}{\int_{0}^{T} \bar{F}_{N}(u) d u+\sum_{n=1}^{N-1}\left(\frac{\mu}{b^{n-1}}(1-p)+p\right) H_{n}(T)+\tau},
$$

where

$$
\int_{0}^{T} \bar{F}_{N}(u) d u=\sum_{i=1}^{N}\left[\prod_{\substack{j=1 \\ i \neq j}}^{n} \frac{a^{j-1}}{a^{i-1}-a^{j-1}}\right]\left(\frac{\lambda}{a^{i-1}}\right)\left[1-\exp \left(-\frac{a^{n-1} t}{\lambda}\right)\right]
$$

In the above equation of the objective function, $T$ is a continuous variable and $N$ is a discrete variable. In this case, assuming $p=0.5$ and using equation (7.1) overpassing numerical calculations, we arrive at $(T, N)^{*}=(301,31)$, such 
that $\mathscr{C}(T, N)$ is minimum at $(T, N)^{*}$ and the long-run average cost is $\mathscr{C}(T, N)=\mathscr{C}(301,31)=-20792$ monetary units. The values of $\mathscr{C}(T, N)$ for $T$ ranging from 0 to 400 time units and $N$ ranging from 0 to 50, for different values of $p$ are evaluated using Maple 6 and the results are plotted in the figures numbered 2 to 7.

When $p=0$, then we obtain $(T, N)^{*}=(41,11)$ with the corresponding $\mathscr{C}(T, N)^{*}=-1623.5022$. When $p=0.3$, then we obtain $(T, N)^{*}=(41,11)$ with the corresponding $\mathscr{C}(T, N)^{*}=-6610.03$. When $p=0.5$, then we obtain $(T, N)^{*}=(261,16)$ with the corresponding $\mathscr{C}(T, N)^{*}=-5276.50$. When $p=0.7$, then we obtain $(T, N)^{*}=(1,11)$ with the corresponding $\mathscr{C}(T, N)^{*}=-1216.36$. When $p=0.9$, then we obtain $(T, N)^{*}=(121,11)$ with the corresponding $\mathscr{C}(T, N)^{*}=-5584.03$. When $p=1.0$, then we obtain $(T, N)^{*}=(21,36)$ with the corresponding $\mathscr{C}(T, N)^{*}=-308.99$.

\section{Comparison of Numerical Results}

In this section, we compare the numerical results evaluated in the previous section.

We concluded that in Section 6 that a bivariate optimal replacement policy $(T, N)^{*}$ for a multistate degenerative system is always better than the univariate replacement polices for the same system.

When $p=0$, that is, when the repair times are non-negligible, then under the univariate $N$ policy, using equation (4.4), we obtain $N^{*}=10$ and $\mathscr{C}\left(N^{*}\right)=12.9169$ monetary units, where as under the bivariate $(T, N)$ policy using equation $(7.1)$, we obtain $(T, N)^{*}=(41,11)$ with the corresponding $\mathscr{C}(T, N)^{*}=-1623.5022$. Therefore it is confirmed that the bivariate $(T, N)$ policy is better than the univariate optimal replacement policy. For other values of $p$ ranging from $p=0$ to $p=1$, the corresponding optimal values of $N^{*},(T, N)^{*}$ and their respective costs are presented in Table 2.

\section{Conclusion}

By considering a repairable system for a monotone process model for a one component multistate degenerative system, explicit expressions for the long-run average cost per unit time under the univariate $T$-policy policy is derived.The conditions for the existence of the optimal replacement policy $T^{*}$ are also derived. Assuming an alternate repair model, for the same system, long-run average cost per unit time under $T$ policy with NNON repair times, $N$ policy with NNON repair times are derived. Also the existence of the optimal replacement polices have been derived. Furthermore we have derived the long run average cost per unit time under a bivariate $(T, N)$ policy with NNON repair times. Also, comparison between the existing $N$-policy and the above polices have been carried out. It is found that the bivariate optimal replacement policy for a multistate degenerative system is better than the univariate policies for the same system. Thangaraj and Rizwan (2001) have established the same result for a two state system with burn-in. Numerical examples are given to illustrate the models and methodology developed in this paper. Also comparison with the existing models have also been carried out. It is concluded here that the bivariate $(T, N)$ policy is the best replacement policy for any multivariate deteriorating system. It is confirmed in this study that the $N$-policy is better than $T$-policy for a monotone process model for a one component multistate degenerative system. This situation triggers us to conjecture that $N$-policy is the best replacement policy among univariate policies for any deteriorating system.

\section{References}

Barlow, R.E. \& F. Proschan. (1965). Mathematical Theory of Reliability, John Wiley and Sons, New York.

Lam, Y. (1988). Geometric Processes and Replacement Problem, Acta. Math. Sinica, 4, 366-377.

Lam, Y. (1990). A Repair Replacement Model, Adv. Appl. Prob., 22, 494-497.

Lam, Y. (2005). A Monotone Process Maintenance odel for a Multistate System, J. App. Prob., 42, 1-14.

Lam, Y., Zhang, Y.L. \& Zheng,Y.H. (2002). A Geometric process equivalent model for a multi-state degenerative system. Europ. J. Oper. Res., 142, 21-29.

Lam, Y., Zhang, Y. L. \& Y. H. Zheng. (2002). A Geometric process equivalent model for a multi-state degenerative system. Europ. J. Oper. Res., 142, 21-29.

Rudin, W. (1976). Principles of Mathematical Analysis, (3rd ed), McGraw-Hill Company, New York.

Stanley, A. D. J. (1993). On Geometric Processes and Repair Replacement Problems,Microelectron. Reliab.,33, 489-491.

Thangaraj, V. \& U. Rizwan. (2001). Optimal Replacement Policies in Burn-in Process for an Alternative Repair Model, Int. J. Info. and Manag. Sci., 12(3), 43-56.

Wang, G. J. \& Y. L. Zhang. (2007). An Optimal Replacement Policy for a Two Component Series System Assuming Geometric Process Repair, Comp. and Math.With App., 54, 192-202.

Zhang, Y. L. (1994). A Bivariate Optimal Replacement Policy for a Repairable System, J. Appl. Prob., 31, $1123-1127$.

Zhang, Y. L. , Yam, R. C. M. \& M.J. Zuo. (2007). A Bivariate Optimal Replacement Policy for a Multistate Repairable System, Reliab. Eng. Syst. Safety., 92, 535-542. 
Table 1. The values of $\mathscr{C}(N)$ for different values of $p$

\begin{tabular}{|c||c|c|c|c|c|}
\hline$N$ & $p=0.0$ & $p=0.3$ & $p=0.5$ & $p=0.7$ & $p=0.9$ \\
\hline 5 & 16.1661 & 15.3032 & 14.4740 & 13.2894 & 11.4581 \\
\hline 6 & 14.6282 & 13.3388 & 12.0699 & 10.2038 & 7.1891 \\
\hline 7 & 13.7135 & 12.1213 & 10.5207 & 8.1039 & 4.0333 \\
\hline 8 & 13.2036 & 11.4003 & 9.5523 & 6.6927 & 1.6778 \\
\hline 9 & 12.9663 & 11.0219 & 8.9936 & 5.7813 & -0.0788 \\
\hline 10 & $\mathbf{1 2 . 9 1 6 9}$ & $\mathbf{1 0 . 8 8 5 6}$ & 8.7312 & 5.2430 & -1.3729 \\
\hline 11 & 12.9987 & 10.9229 & $\mathbf{8 . 6 8 6 9}$ & 4.9891 & -2.2998 \\
\hline 12 & 13.1701 & 11.0854 & 8.8046 & $\mathbf{4 . 9 5 5 2}$ & -2.9284 \\
\hline 13 & 13.4088 & 11.3379 & 9.0427 & 5.0928 & -3.3102 \\
\hline 14 & 13.6883 & 11.6540 & 9.3699 & 5.3645 & $\mathbf{- 3 . 4 8 4 6}$ \\
\hline 15 & 13.9953 & 12.0140 & 9.7619 & 5.7407 & -3.4827 \\
\hline
\end{tabular}

Table 2. Comparison of numerical results between optimal univariate and bivariate polices for different values of $p$

\begin{tabular}{|c||c|c|c|c|c|c|}
\hline$N$ & $p=0.0$ & $p=0.3$ & $p=0.5$ & $p=0.7$ & $p=0.9$ & $p=1.0$ \\
\hline$N^{*}$ & 10 & 10 & 11 & 12 & 14 & 44 \\
\hline $\mathscr{C}(N)^{*}$ & 12.92 & 10.89 & 8.69 & 4.69 & -3.48 & -22.45 \\
\hline \hline$(T, N)^{*}$ & $(41,11)$ & $(41,11)$ & $(261,16)$ & $(11,1)$ & $(121,11)$ & $(36,21)$ \\
\hline $\mathscr{C}(T, N)^{*}$ & -1623.50 & -6610.30 & -5276.50 & -1216.36 & -5584.03 & -308.99 \\
\hline
\end{tabular}

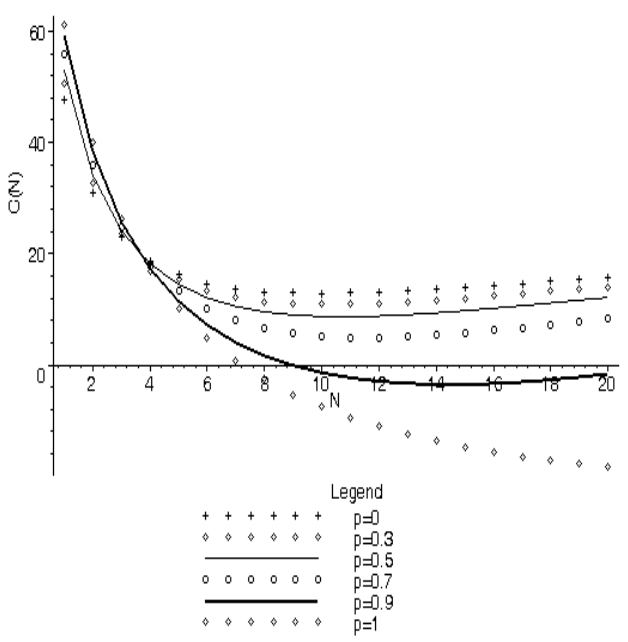

Figure 1. The plots of expected cost $\mathscr{C}(N)$ against $N$ 




Figure 2. The plot of $\mathscr{C}(T, N)$, when $p=0$

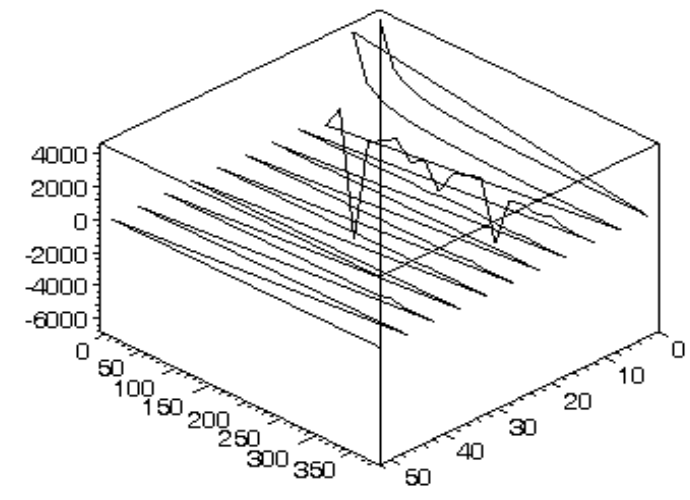

Figure 3. The plot of $\mathscr{C}(T, N)$, when $p=0.3$

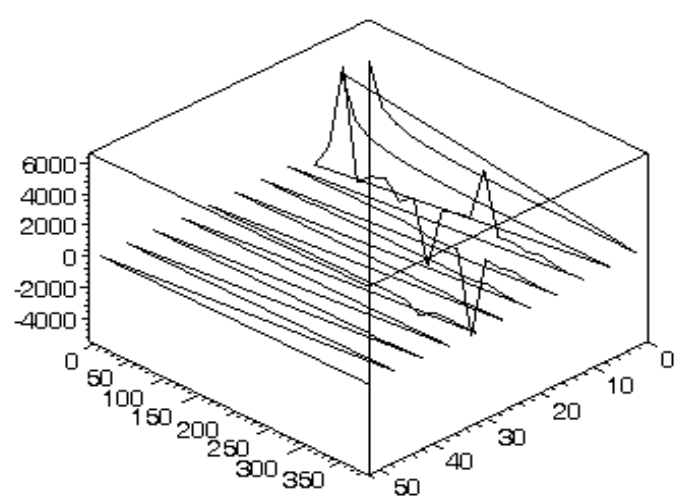

Figure 4. The plot of $\mathscr{C}(T, N)$, when $p=0.5$



Figure 5. The plot of $\mathscr{C}(T, N)$, when $p=0.7$

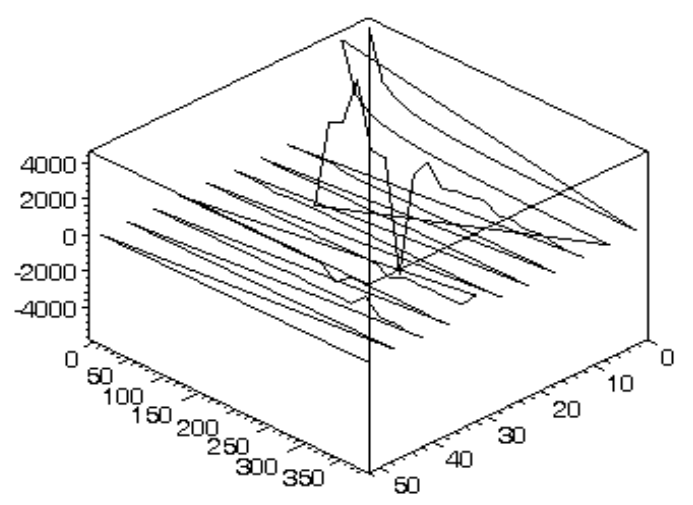

Figure 6. The plot of expected cost $\mathscr{C}(T, N)$, when $p=0.9$

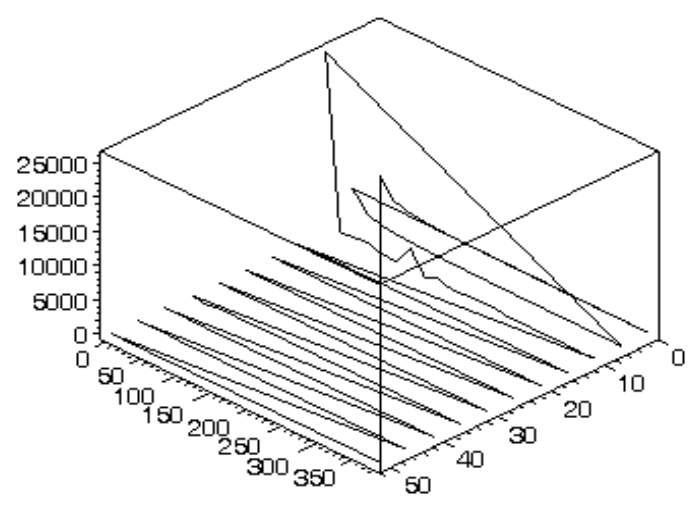

Figure 7. The plot of $\mathscr{C}(T, N)$, when $p=1.0$ 